A General Method to Estimate

Earthquake Moment and Magnitude using Regional Phase Amplitudes

M. E. Pasyanos

November 24, 2009

Bulletin of the Seismological Society of America 
This document was prepared as an account of work sponsored by an agency of the United States government. Neither the United States government nor Lawrence Livermore National Security, LLC, nor any of their employees makes any warranty, expressed or implied, or assumes any legal liability or responsibility for the accuracy, completeness, or usefulness of any information, apparatus, product, or process disclosed, or represents that its use would not infringe privately owned rights. Reference herein to any specific commercial product, process, or service by trade name, trademark, manufacturer, or otherwise does not necessarily constitute or imply its endorsement, recommendation, or favoring by the United States government or Lawrence Livermore National Security, LLC. The views and opinions of authors expressed herein do not necessarily state or reflect those of the United States government or Lawrence Livermore National Security, LLC, and shall not be used for advertising or product endorsement purposes. 


\title{
A general method to estimate earthquake moment and magnitude using regional phase amplitudes
}

Michael E. Pasyanos

Lawrence Livermore National Laboratory

In preparation for BSSA

\begin{abstract}
$\underline{\text { Abstract }}$
This paper presents a general method of estimating earthquake magnitude using regional phase amplitudes, called regional $\mathrm{M}_{\mathrm{o}}$ or regional $\mathrm{M}_{\mathrm{w}}$. Conceptually, this method uses an earthquake source model along with an attenuation model and geometrical spreading which accounts for the propagation to utilize regional phase amplitudes of any phase and frequency. Amplitudes are corrected to yield a source term from which one can estimate the seismic moment. Moment magnitudes can then be reliably determined with sets of observed phase amplitudes rather than predetermined ones, and afterwards averaged to robustly determine this parameter. We first examine in detail several events to demonstrate the methodology. We then look at various ensembles of phases and frequencies, and compare results to existing regional methods. We find regional $\mathrm{M}_{\mathrm{o}}$ to be a stable estimator of earthquake size that has several advantages over other methods. Because of its versatility, it is applicable to many more events, particularly smaller events. We make moment estimates for earthquakes ranging from magnitude 2 to as large as 7. Even with diverse input amplitude sources, we find magnitude estimates to be more robust than typical magnitudes and existing regional methods and might be tuned further to improve upon them. The method yields a more meaningful quantity of seismic moment, which can be recast as $\mathrm{M}_{\mathrm{w}}$. Lastly, it is applied here to the Middle East region using an existing calibration model, but it would be easy to transport to any region with suitable attenuation calibration.

\section{$\underline{\text { Introduction }}$}

This paper presents a general method of estimating earthquake magnitude using regional phase amplitudes, called regional $\mathrm{M}_{\mathrm{o}}$ or regional $\mathrm{M}_{\mathrm{w}}$. There is no shortage of magnitude methods in seismology, nor is there a shortage of methods using regional phases. In fact, Richter's original earthquake magnitude formula (Richter, 1935) makes use of the largest phase recorded at local and regional distances on the horizontal components of the thenstandard Wood-Anderson seismometers with dominant frequencies of around $1 \mathrm{~Hz}$, which is usually the Lg phase. Over the years, a number of magnitude methods and formulas have been developed to take advantage of observed seismic phases (e.g. $\mathrm{m}_{\mathrm{b}}$ for teleseismic body waves, $\mathrm{M}_{\mathrm{S}}$ for teleseismic surface waves) and standard seismic instruments, including a number that utilize regional phase amplitudes. For example, Everden (1967) developed the $\mathrm{m}_{\mathrm{b}}(\mathrm{Pn})$ magnitude to utilize regional Pn observations. Similarly, Nuttli (1973) developed the $\mathrm{m}_{\mathrm{b}}(\mathrm{Lg})$ magnitude for regional $\mathrm{Lg}$ observations. In order to get at moment, Bolt and Herraiz (1983) used the product of the amplitude and 
duration of the S-arrival from Wood-Anderson seismograms to empirically estimate seismic moment by regressing against events with independent moment estimates.

Methods using the regional coda (e.g. Mayeda, 1993; Mayeda et al., 2003) have also been developed to estimate magnitudes. By making use of the averaging power of coda, they have the power to robustly determine magnitudes using a small number of observations, allowing them to be applied to small events. Calibration of the coda, however, is often an involved process that is generally harder to implement than methods using direct phases.

It is widely recognized that moment magnitude methods (e.g. Hanks and Kanamori, 1979) are generally superior to other methods since they capture the low-frequency level that is a better estimator of earthquake size. Also, unlike the nebulous quantity of earthquake magnitude, seismic moment is a physical quantity that is relatable to other parameters through $\mathrm{M}_{\mathrm{o}}=\mu \mathrm{Au}$ where $\mu$ is the shear modulus, $\mathrm{A}$ is the slip area, and $\mathrm{u}$ is the average slip. A reasonable goal then is to use the amplitudes of a variety of regional phases recorded over a potentially broad range of frequencies to estimate the seismic moment and moment magnitude of regional events over a wide magnitude range.

This manuscript is an attempt to generalize the approaches taken to estimate magnitudes using the amplitudes of direct regional phases. This is accomplished by correcting the amplitudes for propagation effects and through the use of an earthquake source model yielding regional estimates of the scalar seismic moment $\mathrm{M}_{\mathrm{o}}$ and regional moment magnitude $\mathrm{M}_{\mathrm{w}}$. One significant advantage to a general method is that it is applicable to a wide list of phases, frequencies, and attenuation structures and that the magnitude can be determined using the data best suited for the problem rather than with predetermined parameters that may not be observed for a given region, epicentral distance, or earthquake size. The result is a straightforward and easy-to-implement general method that encompasses previous regional magnitude methods. In this manuscript, we will explain the regional $\mathrm{M}_{\mathrm{o}}$ approach in detail, show examples of the method applied to individual events and in various combinations for a large dataset of events, and discuss the results.

\section{Approach}

In Pasyanos et al. (2009), we used the amplitudes of regional seismic phases (Pn, Pg, Sn, and $\mathrm{Lg}$ ) to invert for the attenuation structure of the crust and upper mantle in the broad Middle East region. The approach to model the individual amplitudes, which is typical for amplitude tomography, was to parameterize the amplitudes as a product of four terms: the source excitation $\mathrm{S}$, geometrical spreading $\mathrm{G}$, attenuation $\mathrm{B}$, and site effects $\mathrm{P}$. For a given frequency, this is usually represented by the expression:

$$
A_{i j}=S_{i} \quad G_{i j} B_{i j} P_{j}
$$

where $\mathrm{i}$ is the event index and $\mathrm{j}$ is the station index. In the inversion, we assume a geometrical spreading appropriate for the phase, and the phase amplitudes are inverted for attenuation, event source terms, and station site terms. Using a simultaneous multi- 
phase approach (Pasyanos et al., 2009), we used over 11,000 amplitude measurements in the greater Middle East region to invert all of the amplitudes simultaneously and obtain a set of consistent attenuation, source, and site terms. Seismic attenuation is modeled as a set of lateral Qp and Qs values for the crust and another set of lateral Qp and Qs values for the upper mantle. For example, Qs for the crust is plotted as the background in Figure 1. We observe large variations in $\mathrm{Q}$ between high-attenuation (low $\mathrm{Q}$ ) tectonic regions and low-attenuation (high Q) shields and platforms.

In this study, we take the somewhat opposite approach and correct the observed amplitudes of any particular phase and frequency band for the assumed geometrical spreading and the determined attenuation and site effects to estimate the source term.

$$
S_{i}=A_{i j} G_{i j}^{-1} B_{i j}^{-1} P_{j}^{-1}
$$

Once we have the appropriate source term $\left(\mathrm{S}^{\mathrm{P}}\right.$ or $\mathrm{S}^{\mathrm{S}}$, depending on the phase), we can back out the seismic moment $\mathrm{M}_{\mathrm{o}}$ using the same source model that was used in the inversion.

As in our previous study, we used the MDAC formulation (Walter and Taylor, 2001) to tie the source term $S_{i}$ at a particular frequency $\omega$ to the seismic moment $M_{o}$

$$
\begin{aligned}
& \mathrm{S}^{\mathrm{P}}=\mathrm{F}^{\mathrm{P}} \mathrm{M}_{\mathrm{o}} /\left(1+\left(\omega / \omega_{\mathrm{c}}^{\mathrm{P}}\right)^{2}\right) \\
& \mathrm{S}^{\mathrm{S}}=\mathrm{F}^{\mathrm{S}} \mathrm{M}_{\mathrm{o}} /\left(1+\left(\omega / \omega_{\mathrm{c}}\right)^{2}\right)
\end{aligned}
$$

for $\mathrm{P}$-waves and $\mathrm{S}$-waves, respectively. The quantities are related through $\mathrm{F}$, a term related to the radiated energy (specified by equations 4 and 5 in Pasyanos et al., 2009) and, potentially, differing corner frequencies $\omega_{\mathrm{c}}$. This term includes a radiation pattern term which, because the source parameters are not generally known, are set to the average $\mathrm{P}$-wave and $\mathrm{S}$-wave radiation patterns. The corner frequencies are specified as:

$$
\begin{aligned}
& \omega_{\mathrm{c}}{ }^{\mathrm{S}}=\left((\mathrm{K} \sigma) / \mathrm{M}_{\mathrm{o}}\right)^{(1 / 3)} \\
& \omega_{\mathrm{c}}{ }^{\mathrm{P}}=\zeta \omega_{\mathrm{c}}{ }^{\mathrm{S}}
\end{aligned}
$$

where $\mathrm{K}$ is a constant that depends on medium properties, $\sigma$ is the apparent stress, and $\zeta$ is a variable that relates the $\mathrm{P}$ - and $\mathrm{S}$-wave corner frequencies (often set at $\zeta=1$, as was done in the attenuation inversion). While reasonable people can argue over appropriate values for many of these settable parameters, what is important here is that they be set to the same values that were used in the source model of the inversion.

Conceptually, this method bears some similarity to several methods used for surface waves. The first is a study by Stevens and McLaughlin (2001), which uses a source model and Rayleigh wave excitation functions to link the observed spectral amplitudes of 20 second surface waves to the seismic moment $\mathrm{M}_{\mathrm{o}}$ of the event. The second is a study by Russell (2006) which uses a series of narrow-band time-domain surface wave 
amplitudes to estimate the surface wave magnitude $\mathrm{M}_{\mathrm{S}}$ over a wider frequency band (7$30 \mathrm{sec}$ ) generally used for surface waves.

We note here that the source model presented above is only applicable to earthquakes and that other seismic events (e.g. mine blasts, explosions) would not yield consistent values of $\mathrm{M}_{\mathrm{o}}$ between P-waves and S-waves using this model. We actually make use of this discrepancy in high-frequency $\mathrm{P} / \mathrm{S}$ discriminants between earthquakes and nuclear explosions (see Pasyanos and Walter, 2009). In theory, an explosion source model that is applicable to both P-waves and S-waves from explosions (work currently under investigation by a number of research teams) could be used instead to derive $M_{o}$ from the source term $\mathrm{S}$ for these events as well.

\section{Magnitude Estimation}

Using this general procedure, we can theoretically use the amplitude of regional phases in any calibrated frequency band to estimate the seismic moment of an event. In practice, it is likely that magnitude estimates will be calculated at frequencies where the signal-tonoise is good, the attenuation models are reliable, the effect of the radiation pattern is reduced, and the extrapolation to low frequencies is less dependent on model parameters, such as stress drop. To minimize some of these effects, event magnitudes will likely be calculated using a combination of stations, phases, and frequencies.

First, we will consider a couple of events in detail, in order to demonstrate the method. Figure 2 shows an example for an earthquake in the Himalayas recorded at station NIL (Nilore) in Pakistan (see inset). Amplitudes are time-domain RMS amplitudes converted to pseudo-spectral amplitudes to be compatible with the frequency domain method we are using. As indicated by the circles and dashed lines, measured regional amplitudes at this station vary considerably (over three orders of magnitude) across phase and frequency. Amplitudes are only selected when they meet our signal-to-noise criteria (pre-event SNR of 2.0 and pre-signal SNR of 1.0); otherwise, they are not used. When corrected for propagation effects and the source term converted to magnitudes (triangles and solid lines), however, we see great consistency in the results among phases and frequencies. Here, for example, the average $\mathrm{M}_{\mathrm{w}}$ from this station is $6.44 \pm 0.14$, as determined from the mean and standard deviation (mean absolute deviation) of all 21 individual estimates. This compares favorably to a CMT estimate of 6.53 for this event. Network estimates presented here were simply calculated using straight averaging, but maximum likelihood methods (e.g. Ringdal, 1976; McLaughlin, 1988) could also be employed to correct for data censoring near the noise threshold. An alternative approach, not pursued here, is to use the individual amplitude measurements to invert for $\mathrm{M}_{\mathrm{o}}$.

For comparison, a standard $\mathrm{m}_{\mathrm{b}}(\mathrm{Lg})$ would yield a single magnitude data point $(\mathrm{Lg} \sim 1.0$ $\mathrm{Hz})$ as would $\mathrm{m}_{\mathrm{b}}(\mathrm{Pn})(\mathrm{Pn} \sim 1.0 \mathrm{~Hz})$. In the case of the above event, this would yield a single magnitude estimate of 6.55 for $\mathrm{m}_{b}(\mathrm{Pn})$ and 6.41 for $\mathrm{m}_{\mathrm{b}}(\mathrm{Lg})$. Each of these individual measurements, however, would be more susceptible to spectral holes, variable attenuation and phase blockage, and source radiation pattern, all of which could be minimized with averaging. 
Figure 3 shows a second example, an event in the Caucasus recorded at station ABK31, the central element of the Akbulak array in Kazakhstan. This event is considerably smaller than the previous example. Additionally, the path from the source to the receiver (see inset) crosses the Caspian Sea, a path that is known to block Lg (e.g. Baumgardt, 2001). The results can be seen in the amplitude plot. Not only is Lg not recorded above the signal-to-noise in any frequency band, $\mathrm{Pg}$ is only recorded in the 1-2 Hz passband, and $\mathrm{Pn}$ is only recorded above $2 \mathrm{~Hz}$. In other words, if we were relying on making regional magnitude measurements using either $\mathrm{Pn}$ or $\mathrm{Lg}$ around $1 \mathrm{~Hz}$, we would not be able to make any estimate for this event. Using the regional $\mathrm{M}_{\mathrm{o}}$ methodology, however, we are able to make estimates using Sn at a number of frequency bands from 1-10 Hz, as well as Pn from 2-10 Hz, and Pg in the 1-2 Hz passband. The $\mathrm{M}_{\mathrm{w}}$ estimate for this event, as determined from an average of all estimates, is $3.68 \pm 0.14$. In comparison, there was a coda magnitude estimate of 3.78 for this event (Rengin Gok and Eric Matzel, unwritten communication).

\section{Network Magnitudes}

As we have seen in the individual examples, using this general method we could estimate a magnitude at any particular station using a number of combinations of phase and frequency, which could be further combined with other stations to produce a network magnitude. What are the expected advantages of averaging? Averaging among frequencies should minimize narrow spectral effects, like spectral holes, and attenuation calibration effects. Averaging among phases should minimize blockage effects (like Lg blockage), radiation pattern effects (which will be different for P-waves and S-waves) and attenuation calibration effects (which might be better at lower frequencies). Because of the different source-receiver paths, averaging among stations should minimize radiation pattern and attenuation calibration effects. Theoretically, at least, there seems to be an advantage in using multiple estimates, but how well does this work in practice? Are we able to make more magnitude estimates as a result? Does it make a more robust, self-consistent measurement? And is it a better measurement, as determined by comparisons with other estimates of seismic moment?

Included on the map in Figure 1 are 4943 events in the greater Middle East that were considered for magnitude estimates, having at least one regional amplitude measurement recorded at a seismic station passing the signal-to-noise. While a large number of the amplitudes were used in our attenuation tomography, the dataset used here includes many more amplitude measurements from additional stations, more measurements made at existing stations, and from more recent events. For each of these events, we tested various combinations of phases, frequencies, and stations. To baseline our estimates, we selected a combination that most closely resembles the $m_{b}(P n)$ formula (phase $=P n$, frequency band $=1-2 \mathrm{~Hz}$, averaged over stations) and another that most closely resembles the $m_{b}(\mathrm{Lg})$ formula (phase $=\mathrm{Lg}$, frequency band $=1-2 \mathrm{~Hz}$, averaged over stations). For each combination, moments are calculated and a regional network $\mathrm{M}_{\mathrm{w}}$ for the event is determined by averaging the individual $\mathrm{M}_{\mathrm{w}}$ values, accompanied by an associated standard deviation. An $n-1$ term, rather than an $n$ term, is used in the 
denominator of the standard deviation formula to account for the fact that we are estimating the magnitude using the mean, and not comparing values to an independently determined magnitude. This is written as:

$$
\mathrm{s}^{2}=\frac{1}{(\mathrm{n}-1)} \sum_{\mathrm{i}=1}^{\mathrm{n}}\left(\mathrm{x}_{\mathrm{i}}-\overline{\mathrm{x}}\right)^{2}
$$

where $\mathrm{s}^{2}$ is the variance, $\mathrm{x}_{\mathrm{i}}$ are magnitude estimates, $\mathrm{n}$ is the number of estimates, and $\overline{\mathrm{x}}$ is the mean. We use the same signal-to-noise criteria specified earlier. Event magnitudes are only considered if they have more than one magnitude estimate in order to have corresponding estimates of deviation for them.

If we calculate the standard deviation using the combination that closely resembles the $\mathrm{m}_{\mathrm{b}}(\mathrm{Pn})$ formula, we find an average standard deviation of 0.23 magnitude units (m.u.). Using the combination that resembles $\mathrm{m}_{\mathrm{b}}(\mathrm{Lg})$, we find an average standard deviation of 0.16 m.u., and extending this to any phases in the 1-2 Hz passband averaged over stations, we find an average standard deviation of $0.19 \mathrm{~m} . \mathrm{u}$. This is using each phase individually. If all magnitude results from all phases (in the $1-2 \mathrm{~Hz}$ passband) are averaged, then the magnitude standard deviation drops to 0.16 .

For comparison, we have analyzed several catalogs to determine the variation of more typical magnitude estimates. From the ISC bulletin, we determined an average standard deviation of 0.36 for $\mathrm{m}_{\mathrm{b}}$. From the NEIC Earthquake Data Report (EDR) the deviation of $\mathrm{m}_{\mathrm{b}}$ is 0.41 . The average interstation variation in the IDC Reviewed Event Bulletin (REB) is 0.31 for $\mathrm{m}_{\mathrm{b}}, 0.38$ for $\mathrm{M}_{\mathrm{L}}$, and 0.26 for $\mathrm{M}_{\mathrm{S}}$. It appears, then, that the variability of some of the standard formulas using this method appears to be significantly lower. This is likely due to the 2-D variations in the attenuation corrections that can be employed using this technique, rather than relying on a single 1-D attenuation formula. In comparison, the interstation variation of coda magnitudes for earthquakes over broad regions are typically found to be about 0.08 m.u. (Mayeda et al., 2003).

Table 1 shows the results of magnitude analysis for all events shown in the map in Figure 1 using a number of combinations including the ones discussed above. One distinction that should be noted is whether or not a phase or frequency band is listed as specified (i.e. Pn only; Pn and Pg; Pn,Pg,Sn,Lg; etc.) or averaged among all allowable ones. If specified, as in combination 5 (phase $=\mathrm{Pn}, \mathrm{Pg}, \mathrm{Sn}, \mathrm{Lg}$; frequency $=1-2$ ), then we have a number of possible magnitude estimates for each event (e.g. Pn 1-2 Hz, Pg 1-2 Hz, Sn 1-2 Hz, Lg 1-2 Hz). For example, in combination 5, we have 3871 magnitude estimates of 1567 unique events because we have multiple phase estimates for each event. If averaging, as in combination 6 , then all of these are combined into one single estimate. Notice here that we have many more events (4214) since, with phase averaging, we find many more events with two or more individual estimates.

This is perhaps best illustrated by an example. For instance, for a specific event and using Pn 1-2 Hz, if we had only one station estimate, it would not be included as an event under combination 1 , since we are only considering events with more than one estimate. 
Similarly, if we had on one station estimate using Sn 1-2 Hz, it would not appear as an event in combination 3. Because it doesn't make the criteria for either of these, it would also not appear in combination 5. However, it would be included in combination 6 since, averaged, it would have more than one estimate in the 1-2 Hz frequency band for that event.

We have tried a number of combinations (listed in Table 1) and have found some notable trends. As specificity decreases, the number of events (shown in column 4) goes up. This is mainly due to the ability to make amplitude measurements of smaller magnitude events. Using the $\mathrm{m}_{b}(\mathrm{Pn})$-like formula, we were only able to examine 1346 events (down to magnitude 3.2), and using the $\mathrm{m}_{\mathrm{b}}(\mathrm{Lg})$-like formula only 783 events (but down to magnitude 2.8). However, using the broadest combination of any phases and frequency bands, we are able to make 4623 magnitude estimates. The smallest event determined using this method was an event along the Dead Sea Fault and was estimated to have a moment magnitude of 2.04 determined by high-frequency Sn and Lg. GII (Geophysical Institute of Israel) had an $\mathrm{M}_{\mathrm{L}}$ of 1.7 for the event.

While the ability to examine more events is desirable, some combinations can increase the variability compared to combinations of more similar sets. For example, the variability of averaging just $\mathrm{S}$-wave estimates $(0.14)$ is less than $\mathrm{P}$-wave estimates $(0.19)$ or both $\mathrm{P}$ and $\mathrm{S}$ phases in combination $25(0.18)$. Similarly, the variability of just crustal phases Pg and $\operatorname{Lg}(0.14)$ is less than just mantle phases Pn and Sn (0.18) or using all phases (0.15). We see similar trends when comparing low-frequency $(0.5-1,1-2,2-4 \mathrm{~Hz})$ estimates $(0.15)$ and high-frequency $(4-6,6-8,8-10 \mathrm{~Hz})$ estimates $(0.16)$ to ones using frequencies across the whole spectra (0.21). In general, it appears that, all other things being equal, S-wave phases have better consistency than P-wave phases, low-frequencies better than high-frequencies, and averaging over phase (0.18) is better than averaging over frequency $(0.21)$ or averaging over both (0.24). All-in-all, the significantly increased number of events more than offset the slight increases in magnitude variability. This may, in fact, be partially due to the larger numbers of smaller (and noisier) events.

With this dataset, we determined moment magnitudes ranging from $\mathrm{M}_{\mathrm{w}} 2.04-7.14$. This is a significantly larger range (especially at the low end) than the global CMT (and similar methods) and even regional moment tensor solutions. This is due to the fact that this method only requires a few high-frequency regional amplitudes (as opposed to modeling the low-frequency signal). This is comparable to the range of coda magnitude methods. We found that averaging among estimates from different parameters might increase the overall variability compared to estimates of like parameters. This is perhaps not surprising, but the question is whether these more consistent estimates are better estimates? To test this, we compared estimates of regional $\mathrm{M}_{\mathrm{w}}$ to independent $\mathrm{M}_{\mathrm{w}}$ estimates.

Figure 4 shows a comparison of $M_{w}$ s determined from this method with $M_{w} s$ determined using other independent moment estimates, including full moment tensor methods (e.g. global CMT, regional MTs) and coda magnitude methods, which are tied to seismic moment. Variances of the different magnitude combinations are shown in column 6 of 
Table 1. While we generally see differences of about $0.3 \mathrm{~m}$.u., those that involve highfrequency measurements only (combinations 20 and 22) tend to be very high. This is probably due in part to the difficulty of the method to extrapolate small amplitude differences at the high frequencies to consistent variations in the low-frequency spectra, given the large variations in attenuation. This is exacerbated by the fact that the attenuation model is not as well-determined at these frequencies, and is particularly true for the crustal attenuation, since we have significantly fewer amplitude paths for Pg and $\mathrm{Lg}$ at the highest frequencies to provide constraints. We speculate that this is only a empirical limitation and that this can be improved with better calibrations at these frequencies.

One interesting observation is that the RMS of $\mathrm{M}_{\mathrm{w}}$ differences seem to be larger for the more general combinations. It is clear from some of the panels in Figure $\mathbf{4}$ that there is a bias between CMT moments and the moments estimated here. A bias in moments between CMTs and regional moment tensors has been well-documented by Patton (1998) and confirmed by others (e.g. Ghose et al., 1998). It tends to manifest itself as a larger RMS for less-restrictive combinations that record larger number of events over a wide magnitude range. Patton (1998) found that, for Central Asia, CMT moments were larger than regional estimates by an average of 0.27 log-units, which is equivalent to 0.18 magnitude units. We find comparable offsets between CMT moments (mostly the events with $\mathrm{M}_{\mathrm{w}}>5.5$ ) and our moment estimates with less of an offset for the smaller regional moment tensor solutions. The RMS is significantly lower when this bias is removed, but remains high for the high-frequency combinations. The RMS difference with the bias removed in approximately the same for the pseudo- $\mathrm{m}_{\mathrm{b}}(\mathrm{Pn})$, the pseudo- $\mathrm{m}_{\mathrm{b}}(\mathrm{Lg})$, and averaging all phases and frequencies.

\section{$\underline{\text { Discussion }}$}

We have presented a general method for estimating earthquake moment and magnitude using regional phase amplitudes. There are many advantages to the proposed method. The first is that the method outlined is very general. The benefit of its applicability to other phases and frequency bands is that regional moment magnitudes can be calculated where other methods (that are tied to specific phases and frequency bands) would fail. Additionally, the method can benefit from the power of averaging what can be highly variable individual estimates. The result is that we have a method in place for looking at large numbers of events ranging in size from small to big and over seven orders of magnitude in seismic moment. Since it is tied to a source model, it is able to compensate for changes to the corner frequency that complicate many magnitude methods.

A second main advantage is that, unlike many other magnitude formulas, this one is not tied to any particular 1-D attenuation model, which are often only applicable to limited regions. For example, when Priestley and Patton (1997) applied the $\mathrm{m}_{b}(\mathrm{Pn})$ and $\mathrm{m}_{\mathrm{b}}(\mathrm{Lg})$ formulas to central Asia, they could not apply the original formulas as is, but needed to calibrate them specifically for the region. Here, not only 1-D but 2-D variations in the amplitudes due to the earth's attenuation structure can be accounted for and included in the moment estimates. The great advantage to this is the transportability of the method. 
In other words, it should move to other regions (including those with high or low attenuation, regions with phase blockage, etc.) without any adjustments other than the necessary calibration of the attenuation structure, which are becoming increasingly available for regions such as Central Asia (Taylor et al., 2003), the western United States (Phillips and Stead, 2008), the Middle East (Pasyanos et al., 2009), etc. Additionally, the method is not tied to any particular instruments (e.g. Wood-Anderson, WWSSN SP, LP) as are other formulas, including Richter magnitude.

An additional advantage is that the resulting magnitude is not "yet-another-magnitude" formula (e.g. $M_{S}, m_{b}, M_{L}, M_{d}$ ) but, through the source model, yields a moment magnitude $\mathrm{M}_{\mathrm{w}}$, which is tied to the seismic moment. Seismic moment is a physical quantity that is more representative of earthquake size and is relatable to other physical source parameters, such as rupture area and average slip. Based on the magnitude variation of individual measurements, it appears to be a rather robust estimator of earthquake size. Magnitude estimation varies considerably less than that of comparable magnitude measurements like $\mathrm{m}_{\mathrm{b}}, \mathrm{M}_{\mathrm{S}}$, and $\mathrm{M}_{\mathrm{L}}$. In the end, direct waves may not prove to be as robust an estimator as coda waves. But, unlike coda methods, only a minimal calibration is required to implement the method in other regions of the world.

\section{Data and Resources}

Seismic data from station NIL (shown in Figure 2) is available at the IRIS data center (www.iris.edu). Seismic data from station ABK31 (shown in Figure 3) is available from the Kazakhstan National Data Center (http://www.kndc.kz). Figures were prepared using GMT (Wessel and Smith, 1998). Amplitude data from study of Pasyanos et al. (2009). Moment tensor solutions from the Global CMT catalog (www.globalcmt.org).

\section{Acknowledgements}

I thank Bill Walter for his critical review of this manuscript, and Rengin Gok and Eric Matzel for their coda magnitudes. This work was prepared under the auspices of the U.S. Department of Energy by Lawrence Livermore National Laboratory under contract DEAC52-07NA27344. This is LLNL contribution LLNL-JRNL-******.

\section{$\underline{\text { References }}$}

Baumgardt, D.R. (2001). Sedimentary basins and the blockage of Lg wave propagation in the continents, Pure Appl. Geophys., 158, 1207-1250.

Bolt, B.A. and M. Herraiz (1983). Simplified estimation of seismic moment from seismograms, Bull. Seism. Soc. Amer., 73, 735-748.

Evernden, J.F. (1967). Magnitude determinations at regional and near-regional distances in the United States, Bull. Seism. Soc. Amer., 57, 591-639. 
Ghose, S., M.W. Hamburger, and C.J. Ammon (1998). Source parameters of moderatesized earthquakes in the Tien Shan, central Asia from regional moment tensor inversion, Geophys. Res. Lett., 25, 3181-3184.

Hanks, T. C., and H. Kanamori (1979). A moment magnitude scale, J. Geophys. Res., 84, $2348-2350$.

Mayeda, K. (1993). mb $(\mathrm{Lg}$ coda): a stable single station estimator of magnitude, Bull. Seism. Soc. Amer., 83, 851-861.

Mayeda, K., A. Hofstetter, J. O'Boyle, and W.R. Walter (2003). Stable and transportable regional magnitudes based on coda-derived moment-rate spectra, Bull. Seism. Soc. Amer., 93, 224-239.

McLaughlin, K.L. (1988). Maximum-likelihood event magnitude estimation with bootstrapping for uncertainty estimation, Bull. Seism. Soc. Amer., 78, 855-862.

Nuttli, O.W. (1973). Seismic wave attenuation and magnitude relations for Eastern North America, J. Geophys. Res., 78, 876-885.

Pasyanos, M.E., W.R. Walter, and E.M. Matzel (2009). A simultaneous multi-phase approach to determine P-wave and S-wave attenuation of the crust and upper mantle, Bull. Seism. Soc. Amer., 99, 3314-3325, doi:10.1785/0120090061.

Pasyanos, M.E. and W.R. Walter (2009). Improvements to regional explosion identification using attenuation models of the lithosphere, Geophys. Res. Lett., 36, L14304, doi:10.1029/2009GL038505.

Patton, H. J. (1998). Bias in the centroid moment tensor for central Asian earthquakes: Evidence from regional surface wave data, J. Geophys. Res., 103, 26,963-26,974.

Phillips, W.S. and R.J. Stead (2008). Attenuation of Lg in the western US using the USArray, Geophys. Res., Lett., 35, L07307, doi:10.1029/2007GL032926.

Priestley, K.F. and H.J. Patton (1997). Calibration of $\mathrm{m}_{b}(\mathrm{Pn}), \mathrm{m}_{\mathrm{b}}(\mathrm{Lg})$ scales and transportability of the Mo:mb discriminant to new tectonic regions, Bull. Seism. Soc. Amer., 87, 1083-1099.

Richter, C.F. (1935). An instrumental magnitude scale, Bull. Seis. Soc. Amer., 25, 1-32.

Ringdal, F. (1976). Maximum-likelihood estimation of seismic magnitude, Bull. Seism. Soc. Amer., 66, 789-802.

Russell, D.R. (2006). Development of a time-domain, variable-period surface-wave magnitude measurement procedure for application at regional and teleseismic distances, Part I: Theory, Bull. Seism. Soc. Amer., 96, 665 - 677. 
Stevens, J.L. and K.L. McLaughlin (2001). Optimization of surface wave identification and measurement, Pure Appl. Geophys., 158, 1547-1582.

Taylor, S.R., X. Yang, and W.S. Phillips (2003). Bayesian Lg attenuation tomography applied to Eastern Asia, Bull. Seism. Soc. Amer., 93, 795-803.

Walter, W.R. and S.R. Taylor (2001). A revised magnitude and distance amplitude correction (MDAC2) procedure for regional seismic discriminants: theory and testing at NTS, Lawrence Livermore National Laboratory, UCRL-ID-146882 http://www.1lnl.gov/tid/lof/documents/pdf/240563.pdf

Wessel, P. and W.H.F. Smith (1998). New, improved version of Generic Mapping Tools released, EOS Trans. AGU, 79, 579.

$\underline{\text { Authors Affiliations and Addresses }}$

Michael E. Pasyanos

Lawrence Livermore National Laboratory

7000 East Avenue L-046

P.O. Box 808, Livermore, California, 94551 
Tables

Table 1. Assessment of magnitude estimates using the regional Mo method. Under the frequency column, ALL means 0.5-1, 1-2, 2-4, 4-6, 6-8 and 8-10 Hz. The avg function in the phase and frequency columns indicates that items are averaged, while items not in parentheses are used individually. In all cases, magnitudes are averaged by station. The fourth column shows the number of unique events. The fifth column shows the standard deviation of magnitude estimates (in magnitude units) from various combinations of individual measurements (listed in parentheses). The sixth column shows RMS of $\mathrm{M}_{\mathrm{w}}$ differences between the regional $M_{o}$ and independent $M_{w}$ estimates (also in magnitude units) and number of event comparisons.

\begin{tabular}{|l|l|l|l|l|l|}
\hline & Phase & Frequency (Hz) & Events & $\begin{array}{l}\text { SD mag. est. (m.u.) } \\
\text { (nobs) }\end{array}$ & $\begin{array}{l}\text { RMS (m.u.) } \\
\text { (nobs) }\end{array}$ \\
\hline 1 & Pn & $1-2$ & 1376 & $0.23(1376)$ & $0.28(1108)$ \\
\hline 2 & Lg & $1-2$ & 803 & $0.16(783)$ & $0.25(637)$ \\
\hline 3 & Sn & $1-2$ & 960 & $0.16(960)$ & $0.25(755)$ \\
\hline 4 & Pg & $1-2$ & 732 & $0.17(732)$ & $0.27(578)$ \\
\hline 5 & Pn,Pg,Sn,Lg & $1-2$ & 1567 & $0.19(3871)$ & $0.27(3078)$ \\
\hline 6 & avg(Pn,Pg,Sn,Lg) & $1-2$ & 4214 & $0.16(4214)$ & $0.28(2198)$ \\
\hline 7 & Pn & ALL & 1460 & $0.23(5429)$ & $0.35(4406)$ \\
\hline 8 & Lg & ALL & 1270 & $0.16(2365)$ & $0.30(1842)$ \\
\hline 9 & Sn & ALL & 1226 & $0.17(3575)$ & $0.35(2866)$ \\
\hline 10 & Pg & ALL & 1055 & $0.18(2175)$ & $0.33(1719)$ \\
\hline 11 & avg(Pn,Pg) & ALL & 3573 & $0.19(11134)$ & $0.36(6838)$ \\
\hline 12 & avg(Sn,Lg) & ALL & 3452 & $0.14(8835)$ & $0.35(5357)$ \\
\hline 13 & avg(Pn,Sn) & ALL & 3853 & $0.18(13882)$ & $0.37(8017)$ \\
\hline 14 & avg(Pg,Lg) & ALL & 3428 & $0.14(8852)$ & $0.35(4865)$ \\
\hline 15 & avg(Pn,Pg) & avg(ALL) & 4458 & $0.23(4458)$ & $0.32(2318)$ \\
\hline 16 & avg(Sn,Lg) & avg(ALL) & 4329 & $0.21(4329)$ & $0.31(2216)$ \\
\hline 17 & avg(Pn,Sn) & avg(ALL) & 4296 & $0.23(4296)$ & $0.33(2275)$ \\
\hline 18 & avg(Pg,Lg) & avg(ALL) & 3930 & $0.21(3930)$ & $0.29(2072)$ \\
\hline 19 & Pn,Pg,Sn,Lg & avg(0.5-1,1-2,2-4) & 4521 & $0.18(13140)$ & $0.29(7263)$ \\
\hline 20 & Pn,Pg,Sn,Lg & avg(4-6,6-8,8-10) & 3152 & $0.16(6497)$ & $0.50(3459)$ \\
\hline 21 & avg(Pn,Pg,Sn,Lg) & avg(0.5-1,1-2,2-4) & 4654 & $0.20(4654)$ & $0.28(2385)$ \\
\hline 22 & avg(Pn,Pg,Sn,Lg) & avg(4-6,6-8,8-10) & 3318 & $0.22(3319)$ & $0.48(1701)$ \\
\hline 23 & Pn,Pg,Sn,Lg & ALL & 1667 & $0.20(13544)$ & $0.34(10833)$ \\
\hline 24 & Pn,Pg,Sn,Lg & avg(ALL) & 4575 & $0.21(13775)$ & $0.32(7448)$ \\
\hline 25 & avg(Pn,Pg,Sn,Lg) & ALL & 4511 & $0.18(18209)$ & $0.38(9710)$ \\
\hline 26 & avg(Pn,Pg,Sn,Lg) & avg(ALL) & 4686 & $0.24(4686)$ & $0.31(2388)$ \\
\hline
\end{tabular}




\section{Figure Captions}

Figure 1. Map of our study area showing crustal attenuation at $1 \mathrm{~Hz}$ from Pasyanos et al. (2009). The attenuation map is overlain by events (brown circles) scaled by earthquake magnitude.

Figure 2. Amplitudes and estimated moment magnitudes for an event in the Himalayas recorded at station NIL (see map inset). Amplitudes (values on right axis) and moment magnitude (values on left axis) of regional phases ( $\mathrm{Pn}, \mathrm{Pg}, \mathrm{Sn}, \mathrm{Lg}$ ) as a function of frequency. Symbols are only plotted where the amplitude measurements meet the signalto-noise criteria. $\mathrm{M}_{\mathrm{w}}$ from Global CMT is indicated by the thick gray line.

Figure 3. Similar to Figure 1 for an event in the Caucasus recorded at station ABK31 (see map inset). Independent estimate of $\mathrm{M}_{\mathrm{w}}$ for this event from coda magnitudes is indicated by the thick gray line.

Figure 4. A set of comparisons of $\mathrm{M}_{\mathrm{w}}$ values determined using the regional $\mathrm{M}_{\mathrm{o}}$ method to independent $\mathrm{M}_{\mathrm{w}}$ estimates. Solid color symbols indicate $\mathrm{M}_{\mathrm{w}}$ determined using moment tensor methods (global CMT, regional MTs), while open symbols indicate coda magnitude estimates. a) A comparison for Pn in the 1-2 $\mathrm{Hz}$ passband; b) A comparison for $\mathrm{Lg}$ in the 1-2 $\mathrm{Hz}$ passband; c) A comparison for individual phases $\mathrm{Pn}, \mathrm{Pg}, \mathrm{Sn}$, and $\mathrm{Lg}$ (not averaged) in the 1-2 Hz passband; d) A comparison using a combination of all regional phases and passbands. In all panels, solid line indicates linear fit, while dashed line indicates unity.

\section{$\underline{\text { Figures }}$}




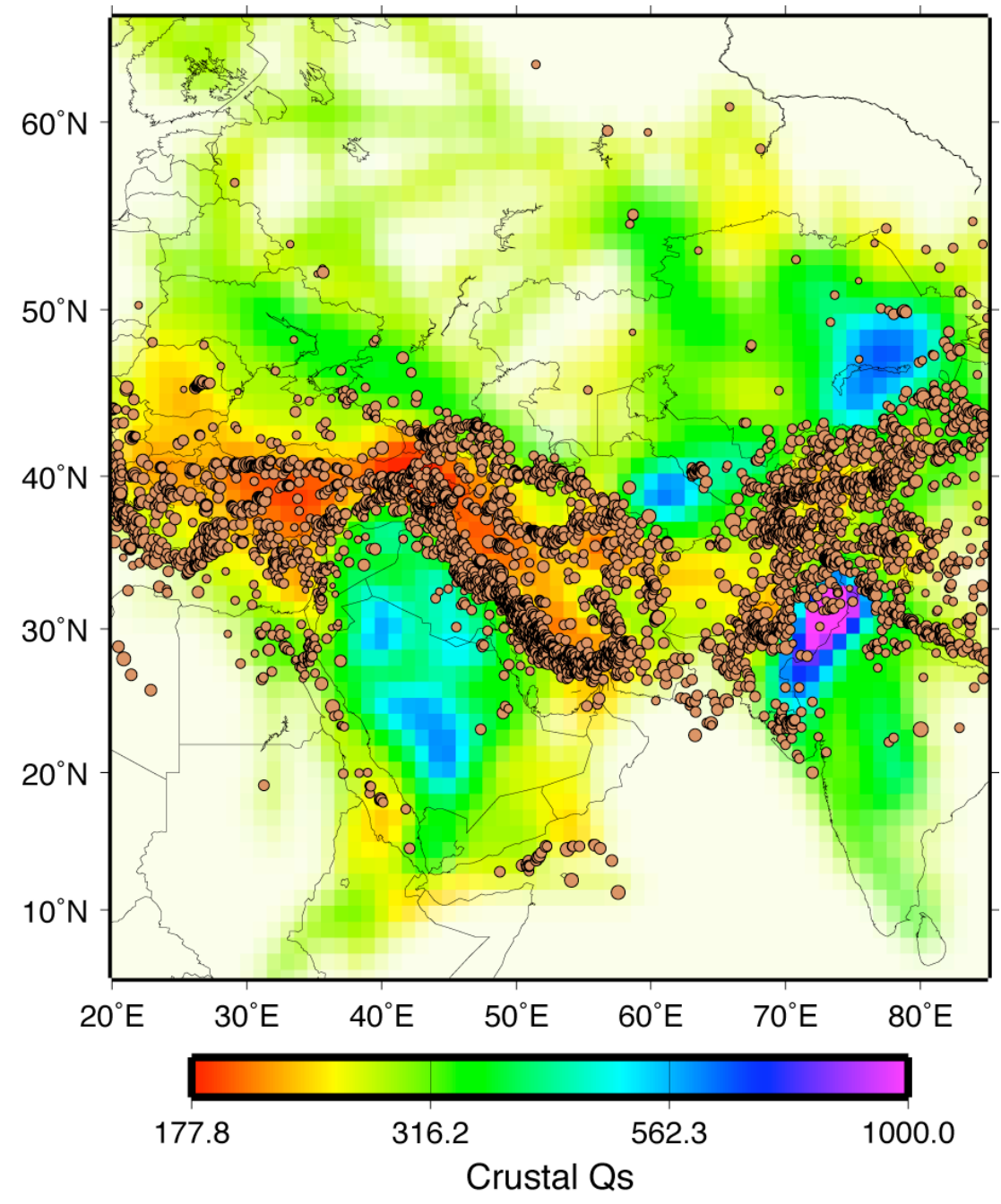

Figure 1 


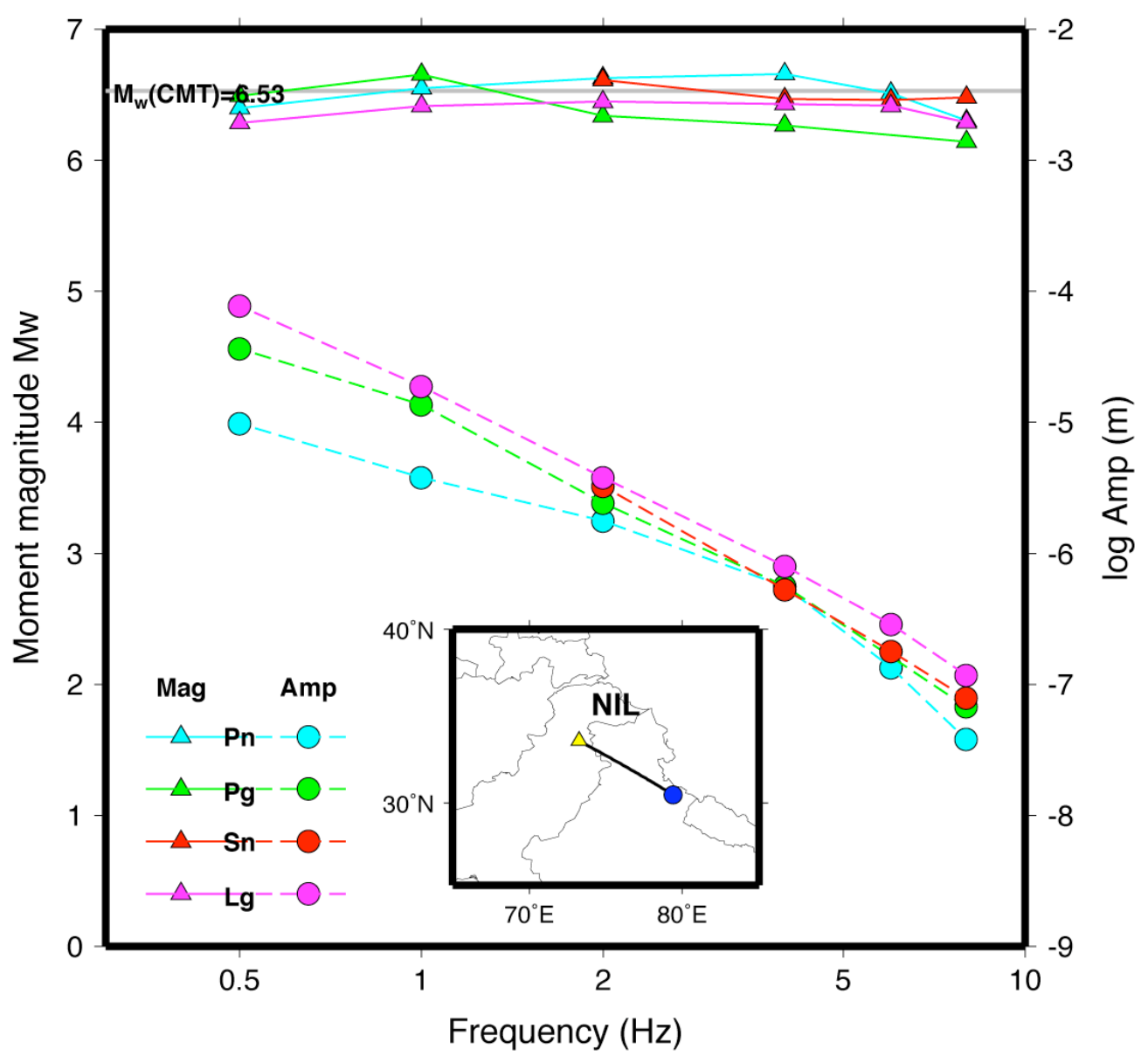

Figure 2 


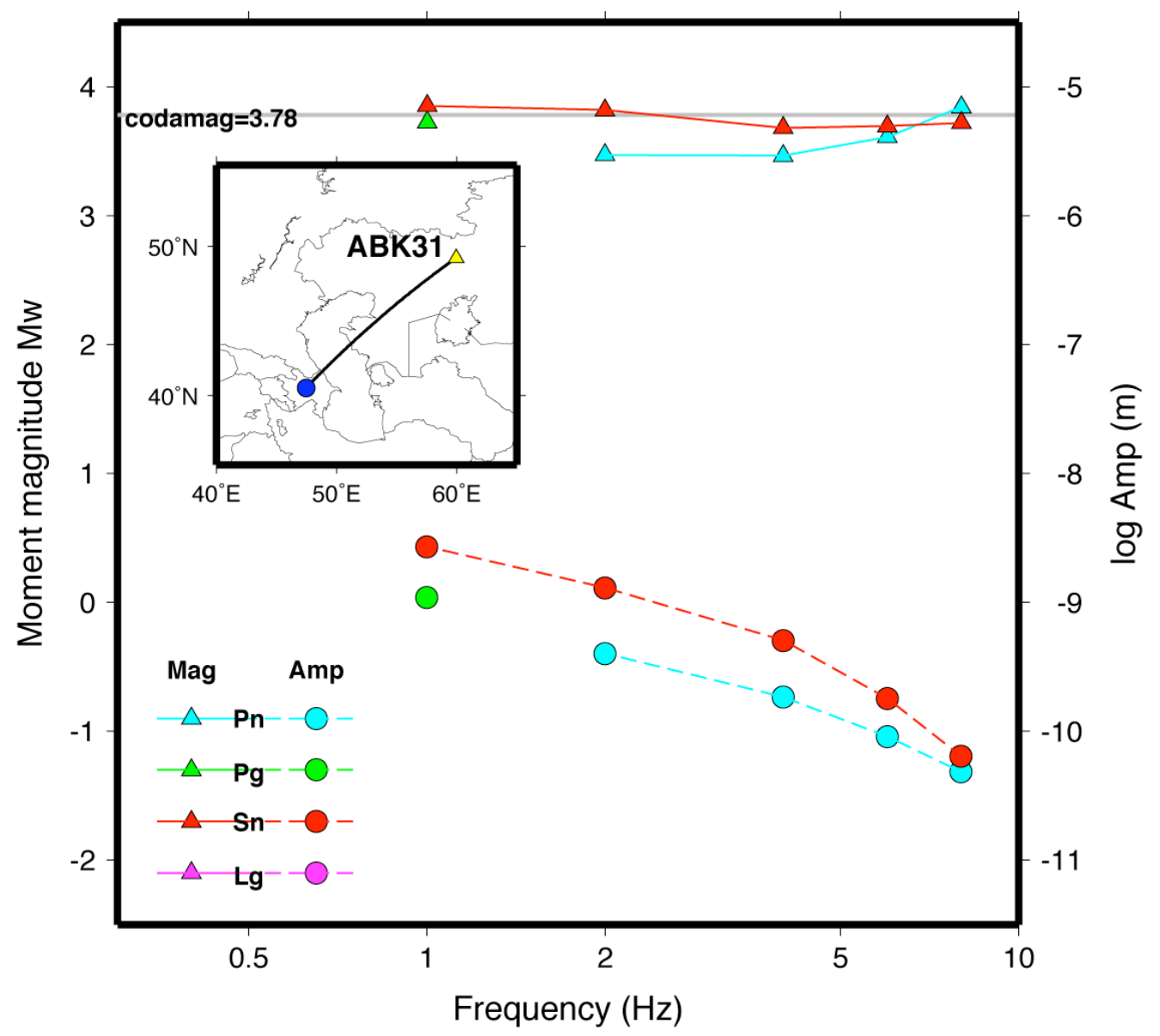

Figure 3 
a)

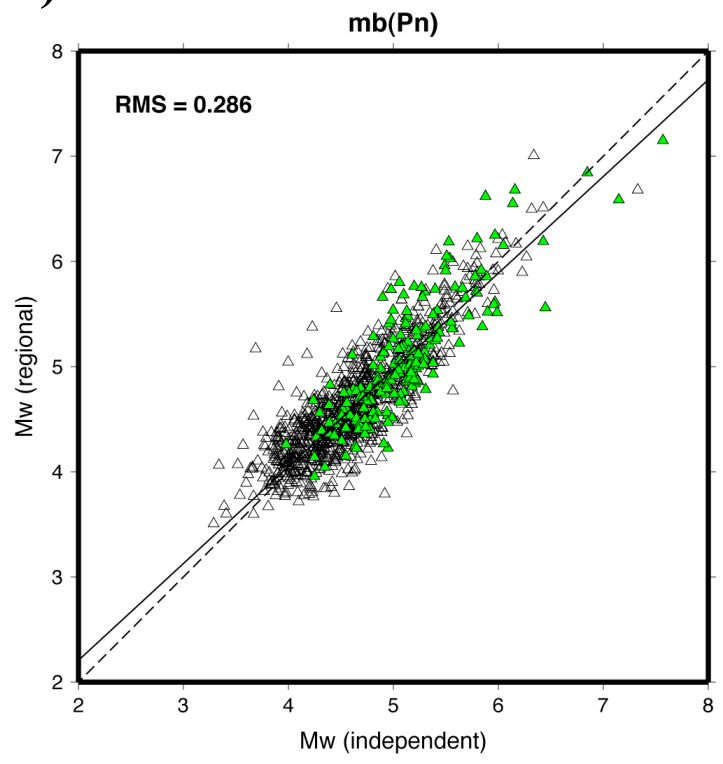

c)

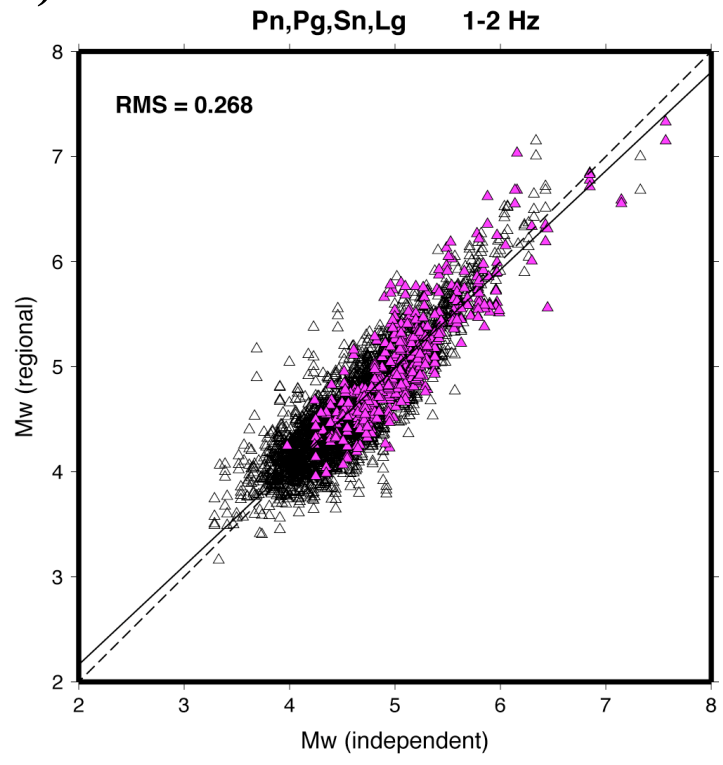

b)

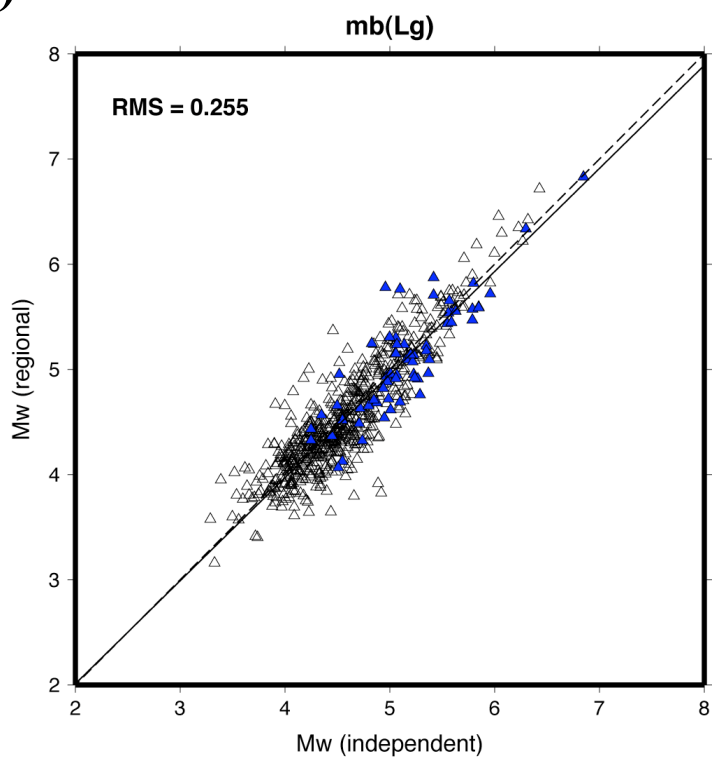

d)

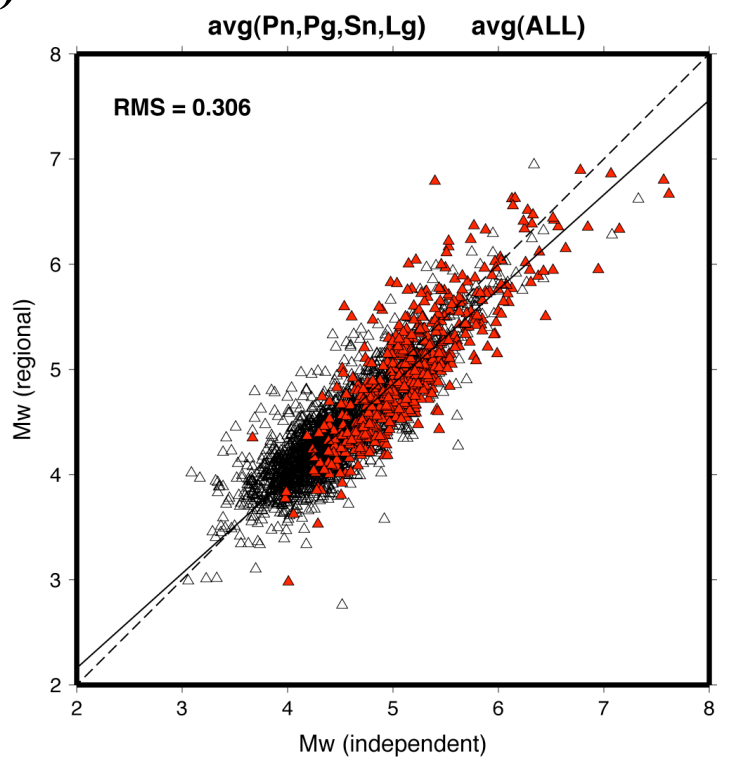

Figure 4 